\title{
The Characterizations of $\left(A_{\mathrm{q}}(U)\right)^{*}$
}

\section{Mark E. Sheingorn**}

(May 17, 1973)

For $q>1$ Bers defines a Banach space $A_{q}(U)=\left\{f \epsilon H(U)\left|\int_{l}\right| f(u) \mid\left(1-|u|^{2}\right)^{q-2} d x d y<\infty\right\}$ and shows that any bounded linear functional $\Lambda$ on $A_{q}(U)$ may be represented as $\Lambda(f)=\int_{l^{\prime}} f(u) \bar{G}(u)(1-$ $\left.|u|^{2}\right)^{2 q-2} d x d y$ where $G \epsilon B_{q}(U)=\left\{h \epsilon H(U)\left|\sup _{u \in l^{\prime}}\right| h(u) \mid\left(1-|u|^{2}\right)^{q}<\infty\right\}$ and is unique. This work is done in $[1]^{1}$. Duren, Romberg, and Shields, pursuant to their work on $H^{p}$ for $p<1$, define a Banach space $B^{p}(p<1)=\left\{f \in H(U)\left|\int_{0}^{2 \pi} \int_{0}^{1}\right| f\left(r e^{i \theta}\right) \mid(1-r)^{1 / p-2} d r d \theta<\infty\right\}$. They show that a bounded linear functional $\Lambda$ on $B^{p}$ may be uniquely represented as $\Lambda(f)=\lim _{r \rightarrow 1} \int_{0}^{2 \pi} f_{r}\left(e^{i \theta}\right) \bar{g}\left(e^{i \theta}\right) d \theta$
where

(i) $f_{r}\left(e^{i \theta}\right)=f_{r}\left(r e^{i \theta}\right)$

(ii) $g \in A=\{h \epsilon H(U) \mid g$ is continuous on $\bar{U}\}$ and $g^{(n-1)}$. and $n-1$ st derivative of $g$, is in $\left.\Lambda_{\alpha}=\left\{h \epsilon H(U) \mid h^{\prime}\left(r e^{i \theta}\right)=0(1-r)^{\alpha-1}\right)\right\}$. (Here $\alpha=1 / p-n$ where $n<1 / p<n+1$, so $\alpha \neq 0$. If $1 / p=n+1$, the conditions on $g$ are: $g \epsilon A, g^{(n-1)} \epsilon \Lambda_{*}=\left\{h \epsilon H(U) \mid h^{\prime \prime}\left(r e^{i \theta}\right)=0\left((1-r)^{-1}\right)\right\}$.) This work appears in [2].

In this paper, after showing that $B^{p} \cong A_{q}(U)$ with $1 / p=q$, we derive the relationship between $G$ and $g$, namely:

$$
G(z)=\sum_{k=0}^{2 n+1} A_{k} \cdot g^{(2 k+1)}(z) \cdot z^{2 k+1},
$$

$(|z|<1)$ where $A_{i}$ are constants, $A_{2 n+1} \neq 0$. (in this case $q=1 / p$ is an integer. The Theorem is slightly different if $q$ is not an integer.)

Key words: Automorphic functions; Hardy spaces.

We begin by showing that $B^{p}$ is isomorphic to $A_{q}=A_{q}(U)$ with $1 / p=q$. (the mapping is the identity.)

Proof: Take $f \in B^{p}$.

$$
\int_{U}|f|\left(1-|u|^{2}\right)^{q-2} d x d y=\frac{1}{2 \pi} \int_{0}^{1}|f|\left(1-r^{2}\right)^{q-2} r d r d \theta \leqslant \frac{\max \left(1,2^{q-2}\right)}{2 \pi} \int_{0}^{2 \pi} \int_{0}^{1}|f|(1-r)^{q-2} d r d \theta .
$$

This shows that $k\|f\|_{B} p \geqslant\|f\|_{A}$. So $B^{p} \subset A_{q}$, with a continuous injection. Now it is easy to verify that if $f \in A_{q}$ then $f \in B^{p}$. Thus we have $i: B^{p} \rightarrow A_{q}, i$ the injection, is continuous and onto. The Open Mapping Theorem implies that $i^{-1}$ is continuous, completing the proof that $B^{p} \cong A_{q}$, with $1 / p=q$.

We turn to the Duren, Romberg and Shields representation. We hereafter will only refer to $A_{q}$, dropping all reference to $B^{p}$. If $f(z)=z^{n}, n \geqslant 0$ (which is certainly in $\mathrm{A}_{q}$ ).

$$
\Lambda\left(z^{n}\right)=\lim _{r \rightarrow 1} \int_{0}^{2 \pi} r^{n} e^{i n \theta} \bar{g}\left(e^{i \theta}\right) d \theta=\int_{0}^{2 \pi} e^{i n \theta} \bar{g}\left(e^{i \theta}\right) d \theta
$$

\footnotetext{
AMS Subiect Classification: 30A58: $30 \mathrm{~A} 78$

*An invited paper. This work was done while the author was a National Academy of Sciences-National Research Council Postdoctoral Research Associate at the National Bureau of Standards, Washington, D.C. 20234

** Present address: Hofstra University. Hempstead, Long Island, N.Y. 11550

' Figures in brackets indicate the literature references at the end of this paper.
} 
since $g \in A$.

But now

$$
\int_{0}^{2 \pi} e^{i n \theta} \bar{g}\left(e^{i \theta}\right)=\lim _{r \rightarrow 1} \int_{0}^{2 \pi} e^{i n \theta} \bar{g}_{r}\left(e^{i \theta}\right) d \theta
$$

by the Lebesgue Dominated Convergence Theorem since $\left\|g_{r}\right\|_{\infty} \leqslant\|g\|_{\infty}$. Now, letting $g(z)=\sum_{k=0}^{\infty} b_{k} z^{k}$,

$$
\begin{aligned}
\int_{0}^{2 \pi} e^{i n \theta} \bar{g}_{r}\left(e^{i n \theta}\right) d \theta & =\int_{0}^{2 \pi} e^{i n \theta} \sum_{0}^{\infty} \bar{b}_{k} r^{k} e^{-i k \theta} d \theta \\
& =\sum_{0}^{\infty} \int_{0}^{2 \pi}\left(\bar{b}_{k} r^{k} e^{i n \theta-i k \theta}\right) d \theta
\end{aligned}
$$

(since the power series for $g_{r}$ converges absolutely in $\bar{U}$ )

$$
=2 \pi \bar{b}_{n} r^{n}
$$

and

$$
\lim _{r \rightarrow 1} \int_{0}^{2 \pi} e^{i n \theta} \bar{g}_{r}\left(e^{i \theta}\right) d \theta=2 \pi \bar{b}_{n}
$$

We have proved

LemMa 1: If $\mathrm{g}(\mathrm{z})=\sum_{\mathrm{k}=0}^{\infty} \mathrm{b}_{\mathrm{k}} \mathrm{z}^{\mathrm{k}}$,

$$
\Lambda\left(\mathrm{z}^{\mathrm{n}}\right)=2 \pi \overline{\mathrm{b}}_{\mathrm{n}} .
$$

Now we turn to the Bers representation of $\Lambda\left(\mathrm{z}^{\mathrm{n}}\right)$. If

$$
\begin{aligned}
\mathrm{G}(\mathrm{z}) & =\sum \mathrm{a}_{\mathrm{k}} \mathrm{z}^{\mathrm{k}}, \lambda(\mathrm{z})=\left(1-|\mathrm{z}|^{2}\right) \\
\Lambda\left(\mathrm{z}^{\mathrm{n}}\right) & =\iint_{\mathrm{U}} \mathrm{z}^{\mathrm{n}} \overline{\mathrm{G}}(\mathrm{z}) \lambda^{2 \mathrm{q}-2} \mathrm{dxdy} \\
& =\frac{1}{2 \pi} \int_{0}^{1} \int_{0}^{2 \pi} \mathrm{r}^{\mathrm{n}} \mathrm{e}^{\mathrm{in} \theta} \sum_{0}^{\infty} \overline{\mathrm{a}}_{\mathrm{k}} \mathrm{r}^{\mathrm{k}} \mathrm{e}^{-\mathrm{ik} \theta}\left(1-\mathrm{r}^{2}\right)^{2 \mathrm{q}-2} \mathrm{rdrd} \theta .
\end{aligned}
$$

Define $G_{\rho}(z)=G(\rho z)$. Then

$$
\sup _{|z|=s}\left|G_{\rho}(z) \lambda^{q}(z)\right|=\sup _{|z|=s}\left|G_{\rho}(z)\right| \lambda^{q}(s)
$$

(since $\lambda$ depends only on $|z|$ )

$$
\begin{aligned}
& \leqslant \sup _{|z|=s}|G(z)| \lambda^{q}(s) \\
& =\sup _{|z|=s}|G(z)| \lambda^{q}(z) .
\end{aligned}
$$


We have shown that

$$
\begin{aligned}
\left|G_{\rho}(z) \lambda^{q}(z)\right| & \leqslant \sup _{|\zeta| \leqslant|z|}\left|G_{\rho}(\zeta) \lambda^{q}(\zeta)\right| \\
& \leqslant \sup _{|\zeta| \leqslant|z|}\left|G(\zeta) \lambda^{q}(\zeta)\right| \leqslant K ;
\end{aligned}
$$

since $G \epsilon B_{q}$. Then,

$$
\lim _{\rho \rightarrow 1} \iint f \bar{G}_{\rho} \lambda^{2 q-2} d x d y=\iint f \bar{G} \lambda^{2 q-2} d x d y
$$

since

$$
\left|f G_{\rho} \lambda^{2 q-2}\right|=\left|f\left(G_{\rho} \lambda^{q}\right) \lambda^{q-2}\right| \leqslant K\left|f \lambda^{q-2}\right|
$$

by above. But since $f \epsilon A_{q}, K\left|f \lambda^{q-2}\right|$ is integrable so (*) holds. Now, as before, we compute $\iint z^{n} \bar{G}_{\rho} \lambda^{2 q-2} d x d y, n \geqslant 0$.

$\iint z^{n} \bar{G}_{\rho} \lambda^{2 q-2} d x d y$

$$
\begin{aligned}
& =\frac{1}{2 \pi} \int_{0}^{1} \int_{0}^{2 \pi} r^{n} e^{i n \theta}\left(\sum_{0}^{\infty} \bar{a}_{k} \rho^{k} r^{k} e^{-i k \theta}\right)\left(1-r^{2}\right)^{2 q-2} r d r d \theta \\
& =\frac{1}{2 \pi} \int_{0}^{1}\left(1-r^{2}\right)^{2 q-2} \cdot r^{n+1} \cdot \int_{0}^{2 \pi} e^{i n \theta}\left(\sum_{0}^{\infty} a_{k} \rho^{k} r^{k} e^{-i k \theta}\right) d \theta d r \\
& =\frac{1}{2 \pi} \int_{0}^{1}\left(1-r^{2}\right)^{2 q-2} r^{n+1} \sum_{0}^{\infty} \int_{0}^{2 \pi} \bar{a}_{k} \rho^{k} r^{k} e^{i n \theta-i k \theta} d \theta d r
\end{aligned}
$$

(since the series for $G_{\rho}$ converges absolutely for $|z|=r \leqslant 1$ )

$$
\begin{aligned}
& =\frac{1}{2 \pi} \int_{0}^{1}\left(1-r^{2}\right)^{2 q-2} r^{n+1} \bar{a}_{n} \rho^{n} r^{n} \cdot 2 \pi d r \\
& =\frac{1}{2 \pi} \cdot 2 \pi \bar{a}_{n} \rho^{n} \int_{0}^{1}\left(1-r^{2}\right)^{2 q-2} r^{2 n+1} d r
\end{aligned}
$$

and taking limit as $\rho \rightarrow 1$ we get

LEMMA 2: If $\mathrm{G}(\mathrm{z})=\sum_{0}^{\infty} \mathrm{a}_{\mathrm{k}} \mathrm{z}^{\mathrm{k}}$

$$
\Lambda\left(\mathrm{z}^{\mathrm{n}}\right)=\overline{\mathrm{a}}_{\mathrm{n}} \cdot \int_{0}^{1}\left(1-\mathrm{r}^{2}\right)^{2 \mathrm{q}-2} \mathrm{r}^{2 \mathrm{n}+1} \mathrm{dr}=\overline{\mathrm{a}}_{\mathrm{n}} \cdot \mathrm{c}_{\mathrm{n}}
$$

where

$$
c_{n}=\int_{0}^{1}\left(1-r^{2}\right)^{2 q-2} r^{2 n+1} d r
$$


Corollary $3: \mathrm{n} \geqslant 0$

$$
2 \pi b_{n}=a_{n} c_{n}
$$

Proof: By taking conjugates, and noting that $c_{n}$ and $2 \pi$ are real.

We shall need the following computation.

LEMMA 4: Let

$$
p^{c} n=\int_{0}^{1} r^{2 n+1}\left(1-r^{2}\right)^{p} d r
$$

then

$($ Here $p \neq-1$.)

$$
p^{c} n=\frac{n ! \Gamma(p+1)}{\Gamma(p+n+2) \cdot 2}
$$

PRoOF: By integration by parts

$$
\begin{aligned}
p^{c} n & =\int_{0}^{1} r^{2 n+1}\left(1-r^{2}\right)^{p} d r \\
& =\frac{1}{p+1} \int r^{2 n-1}\left(1-r^{2}\right)^{p+1} d r \\
& =\frac{n}{p+1}(p+1)^{c}(n-1) .
\end{aligned}
$$

Also, it is easy to compute

$$
p^{c} 0=\frac{1}{2(p+1)}
$$

Using our recursion equation and initial condition, we obtain:

$$
p^{c} n=\frac{n !}{2(p+1) \ldots(p+n+1)} .
$$

Now

$$
p \Gamma(p)=\Gamma(p+1)
$$

So

or

$$
[2(p+1) \ldots(p+n+1)] \Gamma(p+1)=2 \Gamma(p+n+2)
$$

$$
[2(p+1) \ldots(p+n+1)]=\frac{2 \Gamma(p+n+2)}{\Gamma(p+1)} .
$$

Substituting in $(* *)$, we obtain

$$
p^{c} n=\frac{n ! \Gamma(p+1)}{2 \Gamma(p+n+2)} .
$$

We can now prove the basic theorems. First, the case when $q=n+1$, an integer. 
Theorem 5: Let $\mathrm{q}=\mathrm{n}+1$, then

(1) $\mathrm{G}(\mathrm{z})=A_{2_{\mathrm{n}+1}} \mathrm{~g}^{(2 \mathrm{n}+1)}(\mathrm{z}) \cdot \mathrm{z}^{2 \mathrm{n}+1}+\mathrm{A}_{2 \mathrm{n}} \mathrm{g}^{(2 \mathrm{n})}(\mathrm{z}) \cdot \mathrm{z}^{2 \mathrm{n}}+\ldots+\mathrm{A}_{0} \mathrm{~g}(\mathrm{z})(|\mathrm{z}|<1)$ where $A_{\mathrm{i}}$ are constants, $\mathrm{A}_{2 \mathrm{n}+1} \neq 0$.

Proof: $a_{m}=2 \pi b_{m} / c_{m}$ where

$$
\begin{gathered}
c_{m}=(2 q-2)^{c} m=\frac{m ! \Gamma(2 q-1)}{2 \Gamma(2 q+m)} \\
2 q-2=2(n+1)-2=2 n \\
c_{m}=\frac{m ! 2 n !}{(2 n+m+1) ! \cdot 2} .
\end{gathered}
$$

Then

$$
a_{m}=2 \pi b_{m} / c_{m}=\frac{2 \pi b_{m} \cdot 2 \cdot(2 n+m+1) !}{m ! 2 n !}
$$

Now, $\frac{(2 n+m+1) !}{m !}$ is a polynomial of proper degree $2 n+1$. Such polynomial is a linear combination of the following $2 n+2$ polynomials:

$$
\begin{aligned}
C_{0}(m) & =1 \\
C_{1}(m) & =m \\
C_{2}(m) & =m(m-1) \\
C_{3}(m) & =m(m-1)(m-2) \\
\cdot & \\
\cdot & \\
C_{2 n+1}(m) & =m(m-1) \ldots(m-2 n)
\end{aligned}
$$

Thus we have

$$
a_{m}=\frac{4 \pi b_{m}}{2 n !} \cdot \sum_{i=0}^{2 n+1} B_{i} \cdot C_{i}(m)
$$

$\left(B_{i}\right.$ constant, $\left.B_{2 n+1} \neq 0\right)$; then

$$
a_{m} r^{m} e^{i m \theta}=\frac{4 \pi}{2 n !} \sum_{i=0}^{2 n+1} B_{i} C_{i}(m) b_{m} r^{m} e^{i m \theta}
$$

and, if $r<1$

$$
\begin{aligned}
\sum_{m=0}^{\infty} a_{m} r^{m} e^{i m \theta} & =\sum_{m=0}^{\infty} \frac{4 \pi}{2 n !} \cdot \sum_{i=0}^{2 n+1} B_{i} C_{i}(m) \cdot b_{m} r^{m} e^{i m \theta} \\
& =\frac{4 \pi}{2 n !} \sum_{m} \sum_{i} B_{i} \cdot C_{i}(m) b_{m} r^{m} e^{i m \theta} \\
& =\frac{4 \pi}{2 n !} \sum_{i} \sum_{m} B_{i} C_{i}(m) b_{m} r^{m} e^{i m \theta}
\end{aligned}
$$


(since $r<1$ )

Now,

$$
=\frac{4 \pi}{2 n !} \sum_{i} B_{i} \sum_{m=0}^{\infty} C_{i}(m) b_{m} r^{m} e^{i m \theta}
$$

$$
g^{(k)}(z)=\sum_{m=0}^{\infty} C_{k}(m) b_{m} r^{m-k} e^{i(m-k) \theta}
$$

or

$$
g^{(k)}(z)=z^{-k} \cdot \sum_{m} C_{k}(m) b_{m} r^{m} \cdot e^{i m \theta}
$$

or

$$
z^{k} g^{(k)}(z)=\sum_{m} C_{k}(m) b_{m} r^{m} e^{i m \theta}
$$

Hence

$$
\begin{aligned}
G(z) & =\sum_{m=0}^{\infty} a_{m} r^{m} e^{i m \theta} \\
& =\frac{4 \pi}{2 n !} \sum_{i=0}^{2 n+1} B_{i} \cdot g^{(i)}(z) \cdot z^{i} .
\end{aligned}
$$

Now, with $A_{i}=\frac{4 \pi B_{i}}{2 n !}$ we have $(1) \cdot A_{2 n+1} \neq 0$ because $B_{2 n+1} \neq 0$.

We now turn to the case when $q$ is not an integer.

Corollary 6: Let $\mathrm{n}<\mathrm{q}<\mathrm{n}+1$. Then $\mathrm{G} \in \mathrm{B}_{\mathrm{q}}$ may be written

$$
\mathrm{G}(\mathrm{z})=\sum_{\mathrm{i}=0}^{2 \mathrm{n}+1} \mathrm{~A}_{\mathrm{i}} \cdot \mathrm{z}^{\mathrm{i}} \cdot g^{(\mathrm{i})}(\mathrm{z}), \mathrm{A}_{2 \mathrm{n}+1} \neq 0
$$

where not only is $\mathrm{g}^{(\mathrm{n}-1)} \epsilon \Lambda *, \mathrm{~g} \epsilon \mathrm{A}$ but $\mathrm{g}^{(2 \mathrm{n}+1)}=0 \frac{1}{(1-\mathrm{r})^{\mathrm{q}}}$.

(We shall see shortly that $\mathrm{g}^{(\mathrm{n}-1)} \boldsymbol{\epsilon} \Lambda_{*} \Rightarrow \mathrm{g}^{(2 \mathrm{n}+1)}=0\left(1 /(1-\mathrm{r})^{\mathrm{n}+1}\right.$.)

Note that we do not claim that this $g$ is the same as the $g$ associated with $\Lambda$.

Proof: Choose $\beta$ such that $q+\beta=n+1$. $G \epsilon B_{q} . G \epsilon B_{q+\beta}=B_{n+1}$ since $\lambda(z) \leqslant 1$, and $\beta>0$. Apply Theorem 5, getting $G(z)=\sum_{i=0}^{2 n+1} A_{i} z^{i} g^{(i)}(z)$. The proof that $g^{(2 n+1)}=0\left(1 /(1-r)^{q}\right)$ depends on Lemma 7: Say $\mathrm{f} \in \mathrm{H}(\mathrm{U})$,

Then

$$
\left|f\left(r e^{i \theta}\right)\right|<k /(l-r)^{l} .
$$

$$
\left|\mathrm{f}^{\prime}\left(\mathrm{re}^{\mathrm{i} \theta}\right)\right|<\mathrm{k}^{\prime} /(1-\mathrm{r})^{1+1} \text {. }
$$

Proof: Pick $z, r=|z|, r<p<1, C_{\rho}=\{z|| z \mid=\rho\}$. Then

$$
\begin{gathered}
f^{\prime}(z)=\frac{1}{2 \pi i} \int_{C_{\rho}} \frac{f(\zeta)}{|\zeta-z|^{2}} d \zeta \\
\left|f^{\prime}(z)\right| \leqslant \frac{k}{(1-\rho)^{\prime}} \cdot \frac{1}{2 \pi} \cdot \int_{C_{\rho}} \frac{|d \zeta|}{|\zeta-z|^{2}} .
\end{gathered}
$$


We must estimate the last integral

$$
\int_{C_{\rho}} \frac{|d \zeta|}{|\zeta-z|^{2}}=\int_{0}^{2 \pi} \frac{\rho d \theta}{\left|\rho e^{i \theta}-z\right|^{2}}
$$

(where $\zeta=\rho \mathrm{e}^{i \theta}, d \zeta=\rho i e^{i \theta} d \theta$, so $|d \zeta|=\rho d \theta$ ).

Now, let $\rho=(1+r) / 2$. If we set $z^{*}=z / \rho,\left|z^{*}\right|=2 r /(1+r)<1$, when $r<1$. Then.

$$
\begin{aligned}
\int_{0}^{2 \pi} \frac{\rho d \theta}{\left|\rho e^{i \theta}-z\right|^{2}} & =\frac{1}{\rho} \int_{0}^{2 \pi} \frac{d \theta}{\left|e^{i \theta}-\frac{z}{\rho}\right|^{2}} \\
& =\frac{2}{1+r} \int_{0}^{2 \pi} \frac{d \theta}{\left|e^{i \theta}-z^{*}\right|^{2}}
\end{aligned}
$$

Let us look at the last integral.

$$
\begin{aligned}
2 \pi & =\int_{0}^{2 \pi} \operatorname{Re} \frac{e^{i t}+z^{*}}{e^{i t}-z^{*}} d t \quad \text { (Poisson Kernel) } \\
& =\int_{0}^{2 \pi} \operatorname{Re} \frac{\left(e^{i t}+z^{*}\right)\left(\overline{e^{i t}-z^{*}}\right)}{\left|e^{i t}-z^{*}\right|^{2}} d t \\
& =\int_{0}^{2 \pi} \operatorname{Re} \frac{1-\left|z^{*}\right|^{2}+\left(e^{\overline{i t}} z^{*}-e^{i t} \bar{z}^{*}\right)}{\left|e^{i t}-z^{*}\right|^{2}} d t
\end{aligned}
$$

Now $\overline{e^{i t}} z^{*}-e^{i t} \bar{z}^{*}$ is imaginary so

$$
\begin{aligned}
& =\int_{0}^{2 \pi} \frac{1-\left|z^{*}\right|^{2}}{\left|e^{i t}-z^{*}\right|^{2}} d t \\
& =\left(1-\left|z^{*}\right|^{2}\right) \int_{0}^{2 \pi} \frac{d t}{\left|e^{i t}-z^{*}\right|^{2}}
\end{aligned}
$$

Thus

$$
\frac{2}{1+r} \int_{0}^{2 \pi} \frac{d \theta}{\left|e^{i \theta}-z^{*}\right|^{2}}=\frac{2}{1+r} \cdot \frac{2 \pi}{1-\left|z^{*}\right|^{2}}
$$

Now since $G \epsilon B_{q}, n<q<n+1$ we see that

$(* * *)$

$$
(1-r)^{q} \cdot z^{i} \cdot g^{(i)}(z)=0(1)
$$

for $i>2 n+1$.

$$
G(z)(1-r)^{q}=0(1)+(1-r)^{q} g^{2 n+1}(z) \cdot z^{i}
$$

by $(* * *)$. Thus $g^{2 n+1}=0\left(1 /(1-r)^{q}\right)$.

This concludes the proof of Corollary 6 .

We ask the following question: Is every sum of the form

$\sum A_{i} \cdot z^{i} \cdot g^{(i)}(z)$ in $B_{q}$ ? First, if $q$ is an integer, the answer is yes. For then $g^{n-1} \epsilon \Lambda_{*}$ which 
implies

$$
\begin{aligned}
& g^{n+1}=0(1 /(1-r) \\
& g^{n+2}=0\left(1 /(1-r)^{2}\right) \\
& g^{2 n+1}=0\left(\frac{1}{(1-r)^{n+1}}\right)=0\left(\frac{1}{(1-r)^{q}}\right)
\end{aligned}
$$

successively, by Lemma 7.

Thus clearly

$$
\sum_{i=0}^{2 n+1} A_{i} \cdot z^{i} \cdot g^{(i)}(z) \epsilon B_{q}
$$

If $q$ is not an integer, then not every such sum is in $B_{q}$. Only those with $g^{2 n+1}=0\left(1 /(1-r)^{q}\right)$ will be in $B_{q} . g \epsilon \Lambda_{*}$ only implies that $g^{2 n+1}=0\left(1 /(1-r)^{n+1}\right)$, and $0\left(1 /(1-r)^{n+1}\right)$ is a weaker condition than $0\left(1 /(1-r)^{q}\right)$, since $q<n+1$.

The author wishes to thank Jay Stepelman without whom this work could not have begun.

\section{References}

[1] Bers, L., Completeness theorems for Poincaré series in one variable, Proceedings of the international Symposium on Linear Spaces, Jerusalem, (Jerusalem Academic Press-Pergamon Press, 1961), pp. 88- 100.

[2] Duren, P., Romberg, R., Shields, A., Linear functionals on $H^{p}$ with $0<p<1$, to appear.

(Paper 77B3\&4-382) 